\title{
UNIFORMITY FOR WEAK ORDER CONVERGENCE OF RIESZ SPACE-VALUED MEASURES
}

\author{
JUN KAWABE
}

The purpose of the paper is to show that weak order convergence of a net of Dedekind complete Riesz space-valued $\sigma$-measures is uniform over uniformly bounded, uniformly equicontinuous classes of functions. The paper ends with generalizing Ulam's theorem for tightness of positive, finite Borel measures to Riesz space-valued $\sigma$ measures.

\section{INTRODUCTION}

Let $S$ be a completely regular space. Recall that a net $\left\{m_{\alpha}\right\}_{\alpha \in \Gamma}$ of positive finite Borel measures on $S$ is said to weakly converge to a positive finite Borel measure $m$ on $S$ if $\int_{S} f d m_{\alpha} \rightarrow \int_{S} f d m$ for every bounded, continuous, real-valued function $f$ on $S$.

The fact, giving several conditions equivalent to this weak convergence, has been called the Portmanteau Theorem and is among most powerful theorems in the theory of weak convergence of measures; see Topsøe [14, Theorem II.8.1]. Further, it is known that weak convergence of a sequence of positive finite measures on a separable metric space $S$ is uniform over uniformly bounded, equicontinuous classes of functions on $S$; see Rang Rao [11, Theorem 3.1].

The definition of weak order convergence of Riesz space-valued $\sigma$-measures can be introduced naturally. A net $\left\{\mu_{\alpha}\right\}_{\alpha \in \Gamma}$ of Dedekind complete Riesz space-valued $\sigma$-measures on $S$ is said to weakly converge in order to such a $\sigma$-measure $\mu$ on $S$ if $\int_{S} f d \mu_{\alpha}$ converges to $\int_{S} f d \mu$ in order for every bounded, continuous real-valued function $f$ on $S$. In [8, Theorem 7] it has been proved that a version of the Portmanteau Theorem remains valid for a net of Dedekind complete Riesz space-valued $\sigma$-measures on a completely regular space; see also Boccuto and Sambucini [4].

The purpose of the paper is to show that weak order convergence of a net of Dedekind complete Riesz space-valued $\sigma$-measures on a uniform space $S$ is uniform over uniformly

Received 26th October, 2004

Research supported by Grant-in-Aid for General Scientific Research No. 15540162, Ministry of Education, Culture, Sports, Science and Technology, the Japanese Government.

Copyright Clearance Centre, Inc. Serial-fee code: 0004-9727/05 \$A2.00+0.00. 
bounded, uniformly equicontinuous classes of functions on $S$. The paper ends with generalising Ulam's theorem for tightness of positive, finite Borel measures to Riesz spacevalued $\sigma$-measures.

\section{NOTATION AND PRELIMINARIES}

All the topological spaces in this paper are supposed to be Hausdorff. Denote by $\mathbb{R}$ and $\mathbb{N}$ the set of all real numbers and the set of all natural numbers respectively.

In this section, we recall some basic facts on Riesz spaces and Riesz space-valued $\sigma$-measures.

2.1. RIESz SPACES. A Riesz space is said to be Dedekind complete if every non-empty subset that is bounded above has a least upper bound. Every Dedekind complete Riesz space is Archimedean; see Zaanen [20, Theorem 12.3].

Let $V$ be a Riesz space. Given a net $\left\{u_{\alpha}\right\}_{\alpha \in \Gamma}$ in $V$ we write $u_{\alpha} \downarrow u$ to mean that it is decreasing and $\inf _{\alpha \in \Gamma} u_{\alpha}=u$. The meaning of $u_{\alpha} \uparrow u$ is analogous. A net $\left\{u_{\alpha}\right\}_{\alpha \in \Gamma}$ in $V$ is said to converge in order to an element $u$ in $V$ and write $u_{\alpha} \stackrel{o}{\longrightarrow} u$ or $u=\lim _{\alpha \in \Gamma} u_{\alpha}$ if there exists a net $\left\{p_{\alpha}\right\}_{\alpha \in \Gamma}$ in $V$ with $p_{\alpha} \downarrow 0$ such that $\left|u-u_{\alpha}\right| \leqslant p_{\alpha}$ for all $\alpha \in \Gamma$.

In Zaanen [20, Lemma 10.1 and Theorem 10.2] some properties of order convergence are formulated and proved for sequences in a Riesz space, but the analogous properties are also valid for nets and their proofs are all elementary. We collect them in the following proposition for the readers' convenience.

Proposition 1. Let $V$ be a Riesz space. Let $\left\{u_{\alpha}\right\}_{\alpha \in \Gamma}$ and $\left\{v_{\alpha}\right\}_{\alpha \in \Gamma}$ be nets in $V$.

(i) If $u_{\alpha} \downarrow u$, then for any $\alpha_{0} \in \Gamma$ the subnet $\left\{u_{\alpha}\right\}_{\alpha \geqslant \alpha_{0}}$ is also decreasing and $\inf _{\alpha \geqslant \alpha_{0}} u_{\alpha}=u$. The assertion holds for an increasing net.

(ii) If $u_{\alpha} \downarrow u, v_{\alpha} \downarrow v$ and if $a \geqslant 0, b \geqslant 0$ are real numbers, then $a u_{\alpha}+b v_{\alpha}$ $\downarrow a u+b v, u_{\alpha} \vee v_{\alpha} \downarrow u \vee v$ and $u_{\alpha} \wedge v_{\alpha} \downarrow u \wedge v$. The assertion holds for increasing nets.

(iii) If $u_{\alpha} \uparrow u$, then $u_{\alpha} \stackrel{o}{\longrightarrow} u$. Conversely, if $\left\{u_{\alpha}\right\}_{\alpha \in \Gamma}$ is an increasing and order bounded net and $u_{\alpha} \stackrel{\circ}{\longrightarrow} u$, then $u_{\alpha} \uparrow u$. The assertions hold for a decreasing net.

(iv) If $u_{\alpha} \stackrel{\circ}{\longrightarrow} u, v_{\alpha} \stackrel{\circ}{\longrightarrow} v$ and if $a, b$ are real numbers, then $a u_{\alpha}+b v_{\alpha}$ $\stackrel{o}{\longrightarrow} a u+b v, u_{\alpha} \vee v_{\alpha} \stackrel{o}{\longrightarrow} u \vee v$ and $u_{\alpha} \wedge v_{\alpha} \stackrel{o}{\longrightarrow} u \wedge v$.

In this paper, we need the notions of the limes superior and the limes inferior of a net in a Riesz space. Let $\left\{u_{\alpha}\right\}_{\alpha \in \Gamma}$ be an order bounded net in a Dedekind complete Riesz space $V$. Then $x_{\beta}:=\sup _{\alpha \geqslant \beta} u_{\alpha}$ exists in $V$ for each $\beta \in \Gamma$, and a net $\left\{x_{\beta}\right\}_{\beta \in \Gamma}$ is decreasing and bounded below. By the Dedekind completeness of $V$, there is an element $x \in V$ 
such that $x_{\beta} \downarrow x$ and we write $x:=\lim \sup u_{\alpha}$. Similarly, we write $y:=\lim$ inf $u_{\alpha}$, where $y_{\beta}:=\inf _{\alpha \geqslant \beta} u_{\alpha}$ for all $\beta \in \Gamma$ and $y_{\beta} \uparrow y$.

The proof of the following proposition is also elementary.

Proposition 2. Let $\left\{u_{\alpha}\right\}_{\alpha \in \Gamma}$ and $\left\{v_{\alpha}\right\}_{\alpha \in \Gamma}$ be order bounded nets in a Dedekind complete Riesz space $V$. Then $\lim \inf u_{\alpha}$ and $\lim \sup u_{\alpha}$ exist and the following properties hold.

(i) $\liminf u_{\alpha} \leqslant \lim \sup u_{\alpha}$.

(ii) $u_{\alpha} \stackrel{\circ}{\longrightarrow} u$ if and only if $u=\lim \inf u_{\alpha}=\lim \sup u_{\alpha}$.

(iii) $\lim \inf \left(-u_{\alpha}\right)=-\lim \sup u_{\alpha}$ and $\lim \sup \left(-u_{\alpha}\right)=-\lim \inf u_{\alpha}$.

(iv) $\liminf u_{\alpha}+\liminf v_{\alpha} \leqslant \liminf \left(u_{\alpha}+v_{\alpha}\right)$ and $\limsup \left(u_{\alpha}+v_{\alpha}\right) \leqslant \limsup u_{\alpha}$ $+\lim \sup v_{\alpha}$.

(v) If $u_{\alpha} \leqslant v_{\alpha}$ for all $\alpha \in \Gamma$, then $\liminf u_{\alpha} \leqslant \liminf v_{\alpha}$ and $\limsup u_{\alpha}$ $\leqslant \lim \sup v_{\alpha}$.

(vi) If $u_{\alpha} \stackrel{o}{\longrightarrow} u$, then lim inf $\left(u_{\alpha}+v_{\alpha}\right)=u+\lim \inf v_{\alpha}$ and $\lim \sup \left(u_{\alpha}+v_{\alpha}\right)=u$ $+\lim \sup v_{\alpha}$.

Propositions 1 and 2 are used frequently in this paper without mentioning explicitly. See Aliprantis and Burkinshaw [1], Vulikh [16] and [20] for further information on Riesz spaces and properties of order convergence.

2.2. Riesz SPACE-VAlued $\sigma$-Measures. Let $V$ be a Dedekind complete Riesz space. Let $(\Omega, \mathcal{A})$ be a measurable space, that is, $\mathcal{A}$ is a $\sigma$-field of subsets of the non-empty set $\Omega$. Let $\mu: \mathcal{A} \rightarrow V$ be a finitely additive set function. We say that $\mu$ is positive if $\mu(A) \geqslant 0$ for all $A \in \mathcal{A}$. Every finitely additive, positive set function is monotone and finitely subadditive.

In this paper, we need the following notion of the countable additivity that is defined by using only the order structure on the Riesz space; see Wright [18]: A finitely additive, positive set function $\mu: \mathcal{A} \rightarrow V$ is called a $\sigma$-measure if it is $\sigma$-additive in the sense that whenever $\left\{A_{n}\right\}_{n \in \mathbb{N}}$ is a sequence of pairwise disjoint sets in $\mathcal{A}$ then $\mu\left(\bigcup_{n=1}^{\infty} A_{n}\right)=\sup _{n \in \mathrm{N}} \sum_{k=1}^{n} \mu\left(A_{k}\right)$. We emphasise that only measures taking positive values are considered in this note. As in the scalar case, every $\sigma$-measure has the monotone sequential continuity from below and from above, in other words whenever $\left\{A_{n}\right\}_{n \in \mathrm{N}}$ is an increasing (respectively a decreasing) sequence of sets in $\mathcal{A}$ then $\mu\left(\bigcup_{n=1}^{\infty} A_{n}\right)=\sup _{n \in \mathbb{N}} \mu\left(A_{n}\right)$ (respectively $\mu\left(\bigcap_{n=1}^{\infty} A_{n}\right)=\inf _{n \in \mathrm{N}} \mu\left(A_{n}\right)$ ).

In Wright $[17,19]$ a $V$-valued integral with respect to a $\sigma$-measure $\mu$ is constructed and successful analogues of the monotone convergence theorem and the Lebesgue convergence theorem are obtained. We shall use those results freely in this paper. 


\section{UNIFORMITY FOR WEAK ORDER CONVERGENCE}

Let $S$ be a topological space and $V$ a Dedekind complete Riesz space. Denote by $\mathcal{B}(S)$ the $\sigma$-field of all Borel subsets of $S$, that is, the $\sigma$-field generated by the open subsets of $S$. A $\sigma$-measure $\mu: \mathcal{B}(S) \rightarrow V$ is called a $V$-valued $\sigma$-measure on $S$. The following regularity notions for Riesz space-valued $\sigma$-measures on a topological space are needed in this paper.

DEFinition 3: Let $\mu$ be a $V$-valued $\sigma$-measure on $S$.

(i) $\mu$ is said to be $\tau$-smooth if $\mu(G)=\sup _{\beta \in \Lambda} \mu\left(G_{\beta}\right)$ whenever $\left\{G_{\beta}\right\}_{\beta \in \Lambda}$ is an increasing net of open subsets of $S$ with $G=\bigcup_{\beta \in \Lambda} G_{\beta}$.

(ii) $\mu$ is said to be tight if there exist a net $\left\{p_{\beta}\right\}_{\beta \in \Lambda}$ with $p_{\beta} \downarrow 0$ and a net $\left\{K_{\beta}\right\}_{\beta \in \Lambda}$ of compact subsets of $S$ such that $\mu\left(S-K_{\beta}\right) \leqslant p_{\beta}$ for all $\beta \in \Lambda$.

Every $V$-valued $\sigma$-measure $\mu$ on a topological space $S$ with countable basis (in particular, on a separable metric space) is $\tau$-smooth. This follows from the fact that the union of an arbitrary family of open subsets of $S$ coincides with the union of its countable subfamily by the countability of basis of $S$. As to the tightness see Theorem 12 at the end of the paper.

Let us denote by $C(S)$ the space of all bounded, continuous, real-valued functions on $S$. The following is a natural generalisation of the usual notion of weak convergence of positive, finite, real-valued measures on $S$; see [14, page 40].

Definition 4: We say that a net $\left\{\mu_{\alpha}\right\}_{\alpha \in \Gamma}$ of $V$-valued $\sigma$-measures on $S$ weakly converges in order to a $V$-valued $\sigma$-measure $\mu$ on $S$, and write $\mu_{\alpha} \stackrel{w o}{\longrightarrow} \mu$, if for each $f \in C(S)$ we have $\int_{S} f d \mu_{\alpha} \stackrel{\circ}{\longrightarrow} \int_{S} f d \mu$.

Definition 5: (Lipecki [9]) A $V$-valued $\sigma$-measure $\mu$ on $S$ satisfies the countable chain condition if every family $\mathcal{D}$ of pairwise disjoint sets in $\mathcal{B}(S)$ such that $\mu(D) \neq 0$ for all $D \in \mathcal{D}$, is countable.

If $V$ is super Dedekind complete, in other words it is Dedekind complete and every set in $V$ possessing a supremum contains an at most countable subset having the same supremum, then every $V$-valued $\sigma$-measure on $S$ satisfies countable chain condition. A $V$-valued $\sigma$-measure $\mu$ on $S$ is said to be dominated if there exists a finitely additive, positive set function $m: \mathcal{B}(S) \rightarrow \mathbb{R}$ such that $\mu(A)=0$ whenever $A \in \mathcal{B}(S)$ and $m(A)=0$. Every dominated $V$-valued $\sigma$ measure on $S$ also satisfies countable chain condition.

For any subset $A$ of $S, \bar{A}$ denotes the closure of $A, A^{\circ}$ its interior, and $\partial A$ the boundary of $A$, that is, $\partial A:=\bar{A}-A^{\circ}$. For a $V$-valued $\sigma$-measure $\mu$ on $S$, a Borel subset $A$ of $S$ is called a $\mu$-continuity set if $\mu(\partial A)=0$.

The following is a version of the Portmanteau Theorem and was first proved in [4] for a sequence of Dedekind complete Riesz space-valued means on a normal space. For 
the proof of Theorem 6 see $[8$, Theorem 7$]$.

TheOREM 6. (The Portmanteau Theorem) Let $S$ be a completely regular space and $V$ a Dedekind complete Riesz space. Let $\left\{\mu_{\alpha}\right\}_{\alpha \in \Gamma}$ be a net of $V$-valued $\sigma$-measures on $S$ which is uniformly order bounded, that is, there is an element $u \in V$ such that $\mu_{\alpha}(S) \leqslant u$ for all $\alpha \in \Gamma$. Let $\mu$ be a $V$-valued $\sigma$-measure on $S$. Assume that $\mu$ is $\tau$-smooth. Then the following conditions (a) $-(\mathrm{c})$ are equivalent.

(a) $\mu_{\alpha} \stackrel{w o}{\longrightarrow} \mu$.

(b) $\mu(G) \leqslant \liminf \mu_{\alpha}(G)$ for every open subset $G$ of $S$ and $\mu_{\alpha}(S) \stackrel{\circ}{\longrightarrow} \mu(S)$.

(c) $\lim \sup \mu_{\alpha}(F) \leqslant \mu(F)$ for every closed subset $F$ of $S$ and $\mu_{\alpha}(S) \stackrel{\circ}{\longrightarrow} \mu(S)$.

Each of the above conditions implies the condition

(d) $\mu_{\alpha}(A) \stackrel{o}{\longrightarrow} \mu(A)$ for every $\mu$-continuity Borel subset $A$ of $S$.

Further, if $\mu$ satisfies countable chain condition, then all four conditions given above are equivalent.

If $S$ is a uniform space, then we can add the following condition equivalent to conditions (a)-(c).

(e) $\int_{S} f d \mu_{\alpha} \stackrel{o}{\longrightarrow} \int_{S} f d \mu$ for every bounded, uniformly continuous, real-valued function $f$ on $S$.

REMARK 7. If $S$ is a metric space, then the assumption that $\mu$ is $\tau$-smooth is not necessary in Theorem 6; see [15, Theorem I.3.5].

Denote by $U(S)$ the Banach space of all bounded, uniformly continuous real-valued functions on a uniform space $S$ with norm $\|f\|:=\sup _{s \in S}|f(s)|$. We now state and prove our main result.

THEOREM 8. Let $S$ be a uniform space with the uniformity $\mathcal{U}$. Let $V$ be a Dedekind complete Riesz space. Let $\left\{\mu_{\alpha}\right\}_{\alpha \in \Gamma}$ be a uniformly order bounded net of $V$ valued $\sigma$-measures on $S$. Let $\mu$ be a $V$-valued $\sigma$-measure on $S$. Assume that $\mu$ is tight. Let $\mathcal{F}$ be any uniformly bounded, uniformly equicontinuous family of real-valued functions on $S$. If $\mu_{\alpha} \stackrel{w o}{\longrightarrow} \mu$, then $\mu_{\alpha} \stackrel{\text { wo }}{\longrightarrow} \mu$ uniformly on $\mathcal{F}$, in other words

$$
\sup \left\{\left|\int_{S} f d \mu_{\alpha}-\int_{S} f d \mu\right|: f \in \mathcal{F}\right\} \stackrel{o}{\longrightarrow} 0 .
$$

Proof: By the tightness of $\mu$, there are a net $\left\{p_{\beta}\right\}_{\beta \in \Lambda}$ with $p_{\beta} \downarrow 0$ and a net $\left\{K_{\beta}\right\}_{\beta \in \Lambda}$ of compact subsets of $S$ such that

$$
\mu\left(S-K_{\beta}\right) \leqslant p_{\beta}
$$

for all $\beta \in \Lambda$.

Fix $\varepsilon>0$ and $\beta \in \Lambda$. We first claim that there is an open set $D \in \mathcal{U}$ such that

$$
\sup _{s \in K_{\beta}(D)}|f(s)-g(s)| \leqslant 3 \varepsilon
$$


holds for all $f, g \in \mathcal{F}$ satisfying

$$
\sup _{s \in K_{\beta}}|f(s)-g(s)|<\varepsilon,
$$

where $K_{\beta}(D):=\left\{s \in S:(s, t) \in D\right.$ for some $\left.t \in K_{\beta}\right\}$. In fact, there is an open set $D \in \mathcal{U}$ such that $\sup _{f \in \mathcal{F}}|f(s)-f(t)|<\varepsilon$ whenever $(s, t) \in D$. Take functions $f, g \in \mathcal{F}$ such that (3) holds. Let $s \in K_{\beta}(D)$ and take $t \in K_{\beta}$ with $(s, t) \in D$. Then

$$
|f(s)-g(s)| \leqslant|f(s)-f(t)|+|f(t)-g(t)|+|g(t)-g(s)|<3 \varepsilon,
$$

which implies (2).

Next we claim that

$$
\limsup _{\alpha \in \Gamma} \mu_{\alpha}\left(S-K_{\beta}(D)\right) \leqslant p_{\beta} .
$$

Indeed, since $K_{\beta}(D)$ is an open subset containing the compact set $K_{\beta}$, there exists $g \in U(S)$ vanishing on $K_{\beta}$ such that $0 \leqslant g \leqslant 1$ and $g=1$ on $S-K_{\beta}(D)$ (adapt the proof of [7, Proposition 11.5]). The positive $\sigma$-measures $\mu_{\alpha}$ and $\mu$ satisfy $\mu_{\alpha}\left(S-K_{\beta}(D)\right) \leqslant \int_{S} g d \mu_{\alpha}$ and $\int_{S} g d \mu \leqslant \mu\left(S-K_{\beta}\right)$. It follows from $\mu_{\alpha} \stackrel{w o}{\longrightarrow} \mu$ applied to $g \in U(S)$ that $\int_{S} g d \mu_{\alpha} \stackrel{\circ}{\longrightarrow} \int_{S} g d \mu$. Hence, $\underset{\alpha \in \Gamma}{\limsup } \mu_{\alpha}\left(S-K_{\beta}(D)\right) \leqslant \mu\left(S-K_{\beta}\right) \leqslant p_{\beta}$, which establishes (4).

Now, the set of functions $\mathcal{F}$ restricted to $K_{\beta}$ is uniformly bounded and uniformly equicontinuous in $U\left(K_{\beta}\right)$, so that it is a relatively compact subset of $U\left(K_{\beta}\right)$ by the Arzelà-Ascoli theorem. In other words, there is a finite subset $\mathcal{F}_{0}$ of $\mathcal{F}$ such that for any $f \in \mathcal{F}$ there is a function $f_{0} \in \mathcal{F}_{0}$ with $\sup _{s \in K_{\beta}}\left|f(s)-f_{0}(s)\right|<\varepsilon$, and we have $\sup _{s \in K_{\theta}(D)}\left|f(s)-f_{0}(s)\right| \leqslant 3 \varepsilon$ by (2).

Let $f \in \mathcal{F}$ and take $f_{0} \in \mathcal{F}_{0}$ as above. Then

$$
\left|\int_{S} f d \mu_{\alpha}-\int_{S} f d \mu\right| \leqslant \int_{S}\left|f-f_{0}\right| d \mu_{\alpha}+\sup _{h \in \mathcal{F}_{0}}\left|\int_{S} h d \mu_{\alpha}-\int_{S} h d \mu\right|+\int_{S}\left|f_{0}-f\right| d \mu .
$$

Further, if we put $M:=\sup \{\|f\|: f \in \mathcal{F}\}$, then

$$
\begin{aligned}
\int_{S}\left|f-f_{0}\right| d \mu_{\alpha} & \leqslant \int_{\mathcal{S}-K_{\beta}(D)}\left|f-f_{0}\right| d \mu_{\alpha}+\int_{K_{\beta}(D)}\left|f-f_{0}\right| d \mu_{\alpha} \\
& \leqslant 2 M \mu_{\alpha}\left(S-K_{\beta}(D)\right)+3 \varepsilon \mu_{\alpha}(S)
\end{aligned}
$$

and

$$
\begin{aligned}
\int_{S}\left|f_{0}-f\right| d \mu & \leqslant 2 M \mu\left(S-K_{\beta}\right)+\varepsilon \mu(S) \\
& \leqslant 2 M p_{\beta}+\varepsilon \mu(S) .
\end{aligned}
$$


It follows from (5)-(7) that

$$
\begin{aligned}
\sup _{f \in \mathcal{F}}\left|\int_{S} f d \mu_{\alpha}-\int_{S} f d \mu\right| \leqslant & 2 M \mu_{\alpha}\left(S-K_{\beta}(D)\right)+3 \varepsilon \mu_{\alpha}(S) \\
& +2 M p_{\beta}+\varepsilon \mu(S)+\sup _{h \in \mathcal{F}_{0}}\left|\int_{S} h d \mu_{\alpha}-\int_{S} h d \mu\right| .
\end{aligned}
$$

Now an appeal to $\mu_{\alpha} \stackrel{w o}{\longrightarrow} \mu$ gives $\mu_{\alpha}(S) \stackrel{\circ}{\longrightarrow} \mu(S)$ and

$$
\sup _{h \in \mathcal{F}_{0}}\left|\int_{S} h d \mu_{\alpha}-\int_{S} h d \mu\right| \stackrel{\circ}{\longrightarrow} 0
$$

and hence by (4) and (8) we have

$$
\begin{aligned}
\limsup _{\alpha \in \Gamma}\left\{\sup _{f \in \mathcal{F}}\left|\int_{S} f d \mu_{\alpha}-\int_{S} f d \mu\right|\right\} & \leqslant 2 M \limsup _{\alpha \in \Gamma} \mu_{\alpha}\left(S-K_{\beta}(D)\right)+3 \varepsilon \mu(S)+2 M p_{\beta}+\varepsilon \mu(S) \\
& \leqslant 4 M p_{\beta}+4 \varepsilon \mu(S) .
\end{aligned}
$$

Since $p_{\beta} \downarrow 0$, letting $\varepsilon \rightarrow 0$, the proof is complete.

When $V$ is a normed Riesz space, we can introduce another notion of weak convergence of vector measures by using the norm topology of $V$. Recall that a net $\left\{\mu_{\alpha}\right\}_{\alpha \in \Gamma}$ of $V$-valued vector measures on a topological space $S$ weakly converges in norm to a $V$ valued vector measure $\mu$ on $S$ if the net $\left\{\int_{S} f d \mu_{\alpha}\right\}_{\alpha \in \Gamma}$ converges to $\int_{S} f d \mu$ in the norm topology of $V$ for every $f \in C(S)$; see Dekiert [5, Chapter IV]. Then, it follows from [8, Example 9] that Theorem 8 does not necessarily hold for weak norm convergence even in the case of positive vector measures.

Some conditions equivalent to weak norm convergence of vector measures are given in $[5,13]$ for Banach space-valued vector measures or certain Banach lattice-valued positive vector measures. In März and Shortt [10] the Portmanteau Theorem for Banach lattice-valued positive vector measures is shown with respect to a weaker type of weak convergence that is defined by using the weak topology on the Banach lattice.

We end this paper by showing that every weakly $\sigma$-distributive and Dedekind complete Riesz space-valued $\sigma$-measure is tight on any complete separable metric space; see $[3$, Theorem 1.4] for the corresponding result in the case of probability measures, which is called Ulam's theorem. For the proof we need the following supplementary notion. Denote by $\Theta$ the set of all mappings from $\mathbb{N}$ into $\mathbb{N}$.

Definition 9: Let $V$ be a Riesz space. A double sequence $\left\{r_{i, j}\right\}$ in $V$ is called a regulator if it is order bounded and $r_{i, j} \downarrow 0$ for each $i \in \mathbb{N}$, that is, $r_{i, j} \geqslant r_{i, j+1}$ for each $i, j \in \mathbb{N}$ and $\inf _{j \in \mathbb{N}} r_{i, j}=0$ for each $i \in \mathbb{N}$.

The following lemma gives a way to control a sequence of regulators; see Fremlin [6, Lemma 1C], and Riečan and Neubrunn [12, Proposition 3.2.4] for the proof. 
Lemma 10. (Fremlin's Lemma) Let $V$ be a Dedekind complete Riesz space. Let $\left\{r_{i, j}^{n}\right\}(n=1,2, \ldots)$ be a sequence of regulators in $V$. Then for each fixed element $e \in V$ with $e>0$ there is a regulator $\left\{r_{i, j}\right\}$ in $V$ such that, for any $\theta \in \Theta$ one has

$$
e \wedge \sum_{n=1}^{\infty} \sup _{i \in \mathbb{N}} r_{i, \theta(i+n)}^{n} \leqslant \sup _{i \in \mathbb{N}} r_{i, \theta(i+n)} .
$$

Definition 11: A Dedekind complete Riesz space $V$ is said to be weakly $\sigma$-distributive if whenever $\left\{r_{i, j}\right\}$ is a regulator in $V$ then $\inf _{\theta \in \Theta} \sup _{i \in \mathbb{N}} r_{i, \theta(i)}=0$.

The condition of weak $\sigma$-distributivity is satisfied for many concrete Dedekind complete Riesz spaces, for instance, the function spaces $L^{p}(\Omega, \mathcal{A}, m)(0<p \leqslant \infty)$, where $(\Omega, \mathcal{A}, m)$ is any $\sigma$-finite measure space, the spaces $F(X)$ (respectively $B(X)$ ) of all real functions (respectively bounded real functions) on an arbitrary non-empty set $X$, and the corresponding sequence spaces. See [18] and a recent paper [2] for more information on weakly $\sigma$-distributive Riesz spaces.

We are now ready to give a Riesz space version of Ulam's theorem.

THEOREM 12. Let $S$ be a complete separable metric space. Let $V$ be a Dedekind complete Riesz space. Assume that $V$ is weakly $\sigma$-distributive. Then every $V$-valued $\sigma$-measure $\mu$ on $S$ is tight.

PROOF: Let $\left\{s_{k}\right\}_{k \in \mathbb{N}}$ be a countable dense subset of $S$. For each $k, n \in \mathbb{N}, \bar{B}\left(s_{k}, 1 / n\right)$ denotes the closed ball with center $s_{k}$ and radius $1 / n$.

Fix $n \in \mathbb{N}$ for a moment. Put $p_{j}^{n}:=\mu\left(S-\bigcup_{k=1}^{j} \bar{B}\left(s_{k}, 1 / n\right)\right)$ for each $j \in \mathbb{N}$. It follows from $S=\bigcup_{k=1}^{\infty} \bar{B}\left(s_{k}, 1 / n\right)$ that $p_{j}^{n} \downarrow 0$. Put $q_{i, j}^{n}:=p_{j}^{n}$ for each $i, j, n \in \mathbb{N}$. Then $\left\{q_{i, j}^{n}\right\}_{n \in \mathbb{N}}$ is a sequence of regulators in $V$, so that it follows from Fremlin's lemma that there is a regulator $\left\{r_{i, j}\right\}$ in $V$ such that, for any $\theta \in \Theta$ one has

$$
\mu(S) \wedge \sum_{n=1}^{\infty} \sup _{i \in \mathbb{N}} p_{\theta(i+n)}^{n} \leqslant \sup _{i \in \mathbb{N}} r_{i, \theta(i)} .
$$

Fix $\theta \in \Theta$ and put $j_{0}(n):=\min _{i \in \mathbb{N}} \theta(i+n)$ for each $n \in \mathbb{N}$. Then for each $n \in \mathbb{N}$

$$
p_{j 0(n)}^{n} \leqslant \sup _{i \in \mathbb{N}} p_{\theta(i+n)}^{n}
$$

Put $K_{\theta}:=\bigcap_{n=1}^{\infty} \bigcup_{k=1}^{j_{0}(n)} \bar{B}\left(s_{k}, 1 / n\right)$. Then $K_{\theta}$ is a compact subset of $S$. We first show that

$$
\mu\left(S-K_{\theta}\right) \leqslant \sup _{i \in \mathbb{N}} r_{i, \theta(i)}
$$


Indeed, for each $m \in \mathbb{N}$, it follows from (9) and (10) that

$$
\begin{aligned}
\mu\left(\bigcup_{n=1}^{m}\left(S-\bigcup_{k=1}^{j_{0}(n)} \bar{B}\left(s_{k}, 1 / n\right)\right)\right) & \leqslant \mu(S) \wedge \sum_{n=1}^{m} \mu\left(S-\bigcup_{k=1}^{j_{0}(n)} \bar{B}\left(s_{k}, 1 / n\right)\right) \\
& =\mu(S) \wedge \sum_{n=1}^{m} p_{j_{0}(n)}^{n} \\
& \leqslant \mu(S) \wedge \sum_{n=1}^{m} \sup _{i \in \mathbb{N}} p_{\theta(i+n)}^{n} \\
& \leqslant \sup _{i \in \mathbb{N}} r_{i, \theta(i)}
\end{aligned}
$$

so that (11) holds by the monotone sequential continuity of $\mu$.

Put $p_{\theta}:=\sup _{i \in \mathrm{N}} r_{i, \theta(i)}$ for each $\theta \in \Theta$. It follows from the weak $\sigma$-distributivity of $V$ that $\inf _{\theta \in \Theta} p_{\theta}=0$. Since $\Theta$ is ordered and directed upwards by pointwise partial ordering, $\left\{p_{\theta}\right\}_{\theta \in \Theta}$ is a decreasing net in $V$ with $p_{\theta} \downarrow 0$. Thus, the tightness of $\mu$ follows from (11).

\section{REFERENCES}

[1] C.D. Aliprantis and O. Burkinshaw, Positive operators (Academic Press, London, 1985).

[2] E. D'Aniello and J.D.M. Wright, 'Finding measures with given marginals', Quart. J. Math. 4 (2000), 405-416.

[3] P. Billingsley, Convergence of probability measures (John Wiley \& Sons, New York, 1968).

[4] A. Boccuto and A.R. Sambucini, 'The monotone integral with respect to Riesz space-valued capacities', Rend. Mat. Appl. (7) 16 (1996), 255-278.

[5] M. Dekiert, Kompaktheit, Fortsetzbarkeit und Konvergenz von Vectormaßen, (Dissertation) (University of Essen, Duisburg, 1991).

[6] D.H. Fremlin, Topological Riesz spaces and measure theory (Cambridge University Press, Cambridge 1974).

[7] I.M. James, Topological and uniform spaces (Springer-Verlag, New York, 1987).

[8] J. Kawabe, "The portmanteau theorem for Dedekind complete Riesz space-valued measures', in Nonlinear analysis and convex analysis, (W. Takahashi and T. Tanaka, Editors) (Yokohama Publishers, 2004), pp. 149-158.

[9] Z. Lipecki, 'On unique extensions of positive additive set functions', Arch. Math. 41 (1983), 71-79.

[10] M. März and R.M. Shortt, 'Weak convergence of vector measures', Publ. Math. Debrecen 45 (1994), 71-92.

[11] R. Rang Rao, 'Relations between weak and uniform convergence of measures with applications', Ann. Math. Statist. 33 (1962), 659-680.

[12] B. Riečan and T. Neubrunn, Integral, measure, and ordering (Kluwer, Bratislava, 1997).

[13] R.M. Shortt, 'Strassen's theorem for vector measures', Proc. Amer. Math. Soc. 122 (1994), 811-820. 
[14] F. Topøe, Topology and measure, Lecture Notes in Math. 133 (Springer-Verlag, New York, 1970).

[15] N.N. Vakhania, V.I. Tarieladze and S.A. Chobanyan, Probability distributions on Banach spaces (D. Reidel Publishing Company, Dordrecht, 1987).

[16] B.Z. Vulikh, Introduction to the theory of partially ordered spaces (Wolters-Noordhoff, Groningen, 1967).

[17] J.D.M. Wright, 'Stone-algebra-valued measures and integrals', Proc. London Math. Soc. 19 (1969), 107-122.

[18] J.D.M. Wright, 'The measure extension problem for vector lattices', Ann. Inst. Fourier, Grenoble 21 (1971), 65-85.

[19] J.D.M. Wright, 'Measures with values in a partially ordered vector space', Proc. London Math. Soc. 25 (1972), 675-688.

[20] A.C. Zaanen, Introduction to operator theory in Riesz spaces (Springer-Verlag, Berlin, 1997).

Department of Mathematics

Faculty of Engineering

Shinshu University

4-17-1 Wakasato

Nagano 380-8553

Japan

e-mail: jkawabe@shinshu-u.ac.jp 